\title{
Combustion of hydrogen-oxygen mixture in electrochemically generated nanobubbles
}

\author{
Vitaly B. Svetovoy, ${ }^{1, *}$ Remko G. P. Sanders, ${ }^{1}$ Theo S. J. Lammerink, ${ }^{1}$ and Miko C. Elwenspoek ${ }^{1,2}$ \\ ${ }^{1}$ MESA $^{+}$Research Institute, University of Twente, PO 217, NL-7500 AE Enschede, The Netherlands \\ ${ }^{2}$ FRIAS, University of Freiburg, D-79104 Freiburg, Germany
}

(Received 4 March 2011; revised manuscript received 23 May 2011; published 22 September 2011)

\begin{abstract}
Ignition of exothermic chemical reactions in small volumes is considered as difficult or impossible due to the large surface-to-volume ratio. Here observation of the spontaneous reaction is reported between hydrogen and oxygen in bubbles whose diameter is smaller than a threshold value around $150 \mathrm{~nm}$. The effect is attributed to high Laplace pressure and to fast dynamics in nanobubbles and is the first indication on combustion in the nanoscale. In this study the bubbles were produced by water electrolysis using successive generation of $\mathrm{H}_{2}$ and $\mathrm{O}_{2}$ above the same electrode with short voltage pulses in the microsecond range. The process was observed in a microsystem at current densities $>1000 \mathrm{~A} / \mathrm{cm}^{2}$ and relative supersaturations $>1000$.
\end{abstract}

Below a certain size, material properties can change drastically [1]. For example, the surface tension can support metastable phases of nanocrystals [2] that exist only at high pressure for bulk materials. In liquids the surface tension results in significant pressure inside of nanobubbles. Only long-lived nanobubbles, for which the stability mechanism is still under discussion [3], have been directly observed [4]. It is known that gas-phase reactions in bubbles can be driven by acoustic [5] or hydrodynamic [6] cavitation, but there are no reports on spontaneous chemical reactions. Moreover, ignition of exothermic reactions in small volumes is considered as difficult or not possible at all due to significant heat losses on the volume boundary [7]. In this paper it is demonstrated that, in nanobubbles containing $\mathrm{H}_{2}$ and $\mathrm{O}_{2}$ gases in amounts close to the stoichiometric ratio, the reaction starts spontaneously in bubbles whose size is smaller than a threshold value of $100-200 \mathrm{~nm}$. A simple way to produce such nanobubbles is electrolysis of water with short voltage pulses of alternating polarity. We observed that for pulses shorter than $25 \mu$ s the bubble production disappears unexpectedly accompanied by a considerable damage of the electrode surface. At the same time the short pulses of single polarity still produce gases $\left(\mathrm{H}_{2}\right.$ or $\mathrm{O}_{2}$ ) in agreement with the Faraday law.

For the experiments we used $12 \times 12 \mathrm{~mm}^{2}$ chips $(\mathrm{BF}-33$ glass) containing 16 pairs of microelectrodes of different shapes and sizes, covered with SU8 for electrical insulation [Fig. 1(a)]. The electrodes materials were Pt, Pd, W, and $\mathrm{Au}$ deposited up to $100 \mathrm{~nm}$ on separate substrates and patterned. The solution for electrolysis was $1 \mathrm{M}$ of $\mathrm{Na}_{2} \mathrm{SO}_{4}$ in deionized water. The process was observed with a homemade stroboscopic system [8], which controlled the electric and light pulses on the scale of microseconds. The threshold voltage $U_{0}$ for gas production was high $(2.5-4 \mathrm{~V})$ as often happens in microsystems $[9,10]$, but it is not related to high electrochemical overpotential. $U_{0}$ can be significantly reduced by treatment of the samples in $\mathrm{O}_{2}$ plasma and is not very sensitive to the separation between electrodes. The potential drops mainly over the surface films deposited on the electrodes during fabrication (hydrocarbons).

*V.Svetovoy@utwente.nl
In water electrolysis $\mathrm{H}_{2}$ and $\mathrm{O}_{2}$ microbubbles have been produced on a timescale of milliseconds [9,11]. We tested the process at short times of $10-100 \mu \mathrm{s}$ by applying negative or positive voltage pulses to the working electrode (the other one was grounded) and producing hydrogen or oxygen above this electrode. Clear visible contrast ("haze") was observed due to the gas formation, but separate bubbles were not resolved for at least $t \leqslant 30 \mu$ s (see the first figure in the Supplemental Material [12]). One can conclude that, in this period, the gas existed mainly in the form of nanobubbles and dissolved molecules. For $30 \mu \mathrm{s} \mathrm{H}_{2}$ and $\mathrm{O}_{2}$ molecules can diffuse from the electrode on distances of 370 and $250 \mathrm{~nm}$, respectively. This diffusion length is the upper limit for the bubble diameter. The perimeter of the electrodes is the preferred place for gas nucleation, but the gas is also formed above the inner regions signaling the homogeneous nucleation of bubbles [13]. Due to the short time scale, we were not able to directly observe small nanobubbles [e.g., with an atomic force microscope (AFM) $[4,14]]$ that do not contribute to the light scattering. During the first 100-300 $\mu$ s the nanobubbles grow and aggregate forming microsized bubbles, but subsequently, evolution becomes very slow (see the second figure of Supplemental Material [12]). The deflation of the bubbles is a slow process [10,15], which prevents application of electrolysis for fast reversible phase transitions in microactuators, pumps, or valves $[9,10,16]$, for example.

In order to analyze the chemical reaction, one has to create bubbles containing both gases. To achieve this we applied square voltage pulses of alternating polarity repeated with a frequency $f$ so that hydrogen and oxygen were produced alternately above the working electrode. The volume of gas in this case was expected to be larger than the volume of $\mathrm{H}_{2}$ or $\mathrm{O}_{2}$ produced by the pulses of the same frequency and amplitude but with a fixed polarity. The experiment demonstrated that this was the case for $f<10-20 \mathrm{kHz}$, but, at higher frequencies, the gas volume produced by pulses of alternating polarity was drastically decreased (Fig. 1). The effect of gas disappearance is related to its stoichiometric production. Deviation of the duty cycle of the pulses from $50 \%$ in any direction leads to an increase in the visible gas volume (see the third figure in Supplemental Material [12]) revealing excessive $\mathrm{H}_{2}$ or $\mathrm{O}_{2}$.

Electrolysis with alternating polarity pulses results in a significant modification of the electrode surface that is clearly 

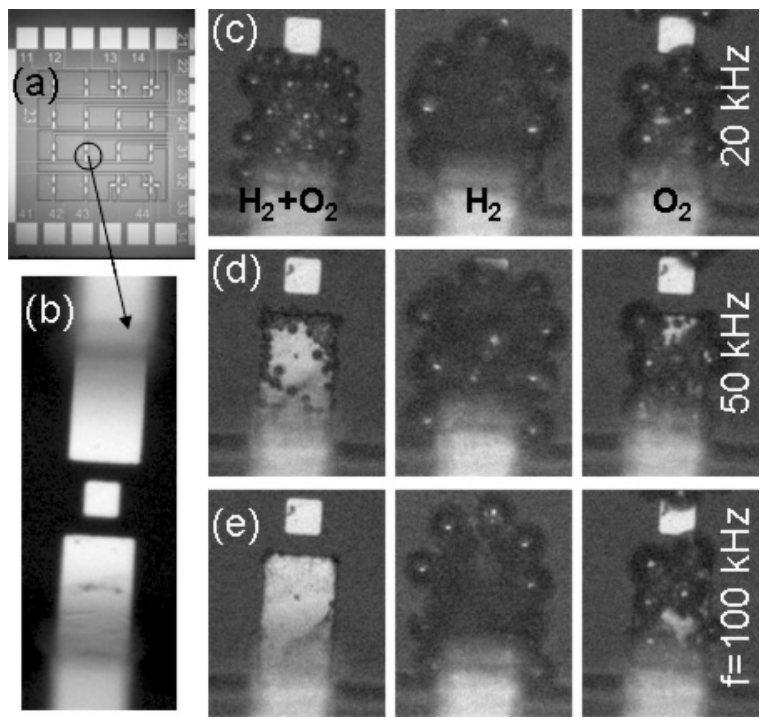

FIG. 1. Disappearance of gas production for alternating polarity pulses. (a) Chip. (b) Pt electrodes before the process. (c) (Row) Gas production after $1 \mathrm{~ms}$ process. The left image is for alternating sign voltage pulses ( $U=4.5 \mathrm{~V}, f=20 \mathrm{kHz}$ ), both $\mathrm{H}_{2}$ and $\mathrm{O}_{2}$ gases are produced. The middle image is for negative voltage pulses of the same $U$ and $f$, only $\mathrm{H}_{2}$ is produced. The right image is for positive voltage pulses, only $\mathrm{O}_{2}$ is produced. (d) The same as (c), but for $f=50 \mathrm{kHz}$, a small amount of gas is visible in the left image. (e) The same as (c), but for $f=100 \mathrm{kHz}$, no gas is visible in the left image.

visible in an optical microscope [Fig. 2(a)]. This effect has never been observed when fixed polarity pulses are used. The modification becomes stronger with the process time. We call this effect "wear of electrodes." It was observed for all electrode materials but was strongest for gold and weakest for tungsten, correlating with the material's yield strength. We established that the light wear resembles debris that can be swept away by production of one kind of gas with fixed polarity pulses.

More detailed information regarding the electrode modification can be extracted from AFM scans as shown in Fig. 2. Significant displacement of the electrode material is visible. From comparison of the images in Figs. 2(c) and 2(d) it is clear that the process changes the structure of the rough surface. We concluded that the surface modification has a mechanical origin. We can exclude the idea of a chemical origin for the wear as the effect has been observed with a range of electrode materials. An electrochemical origin for the wear is also impossible, as the effect has not been observed for single polarity pulses.

To gain a deeper insight into the disappearance of gas production, we observed the process with a vibrometer (Polytec MSA-400). Some results are shown in Fig. 3. A laser beam $(\lambda=633 \mathrm{~nm})$, with a diameter of $1.5 \mu \mathrm{m}$, was focused on the electrode at some distance (around $5 \mu \mathrm{m}$ ) from the edge. The signal $v(t)$ (velocity) is nonzero because the current results in gas formation, which changes the refraction index of the liquid (possible thermal effect can be neglected [12]). This is true so long as the gas does not form bubbles comparable in size with $\lambda / \pi$; otherwise scattering on the bubbles becomes overwhelming, and the signal cannot
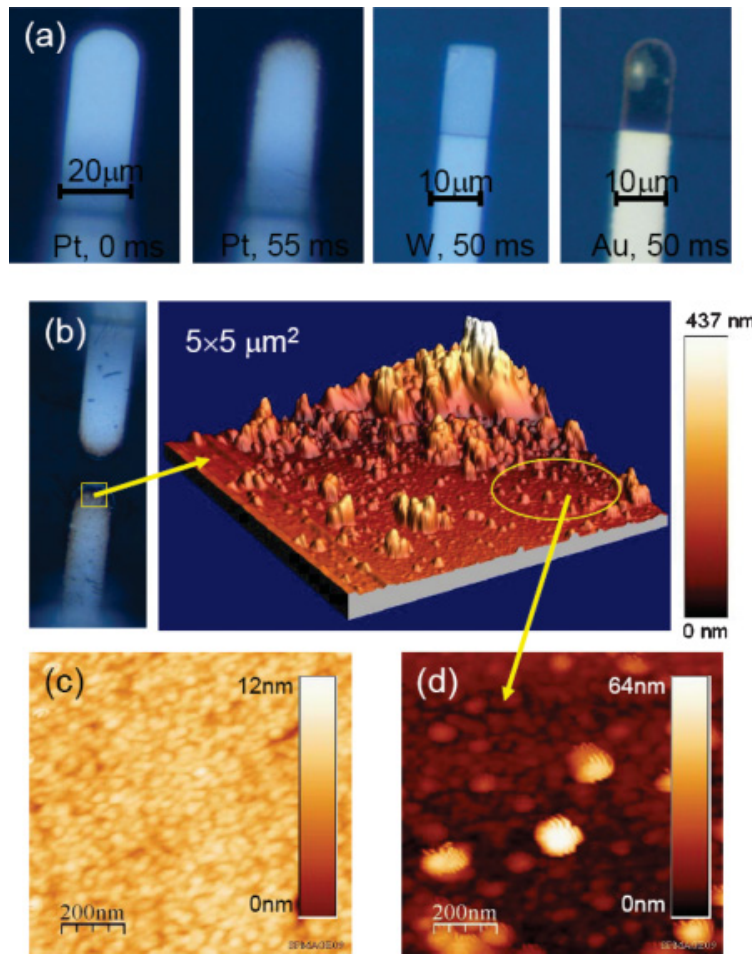

FIG. 2. (Color) Modification (wear) of the electrode surface with the process, $U=4.5 \mathrm{~V}, f=100 \mathrm{kHz}$. (a) Electrodes of different materials in liquid after a fixed process time. The left image is a Pt electrode before the process (reference). (b) The optical image in air (left) of the well-worn Pt electrodes $(55 \mathrm{~ms})$. The right image is an AFM (Veeco PicoForce) scan made somewhere in the highlighted region. A significant displacement of the electrode material is visible. (c) Pt electrode before the process (reference). (d) Zoomed area image demonstrates significant change in the surface roughness.

be related to the amount of gas in the liquid [as seen in Fig. 3(a)]. When the bubbles are small enough, the signal unambiguously demonstrates a response to the current pulses [Fig. 3(b)]. Note that vibrations do not contribute to $v(t)$ : The signal is zero everywhere except of the open part of the electrode.

We can integrate the signal $v(t)=-\mathrm{d}(\Delta d) / \mathrm{d} t$ to find the change in the optical path $\Delta d(t)$ as shown in Fig. 3(c) (see also the fourth figure in Supplemental Material [12]). If it is assumed that, within the laser spot, the gas production is homogeneous, then $\Delta d(t)$ must be a monotonous function of time. The observed nonmonotonous character of $\Delta d(t)$ clearly indicates the presence of a reaction between the gases, which periodically reduces the number of gas molecules in the system. Reaction in bulk between dissolved molecules is negligible. It is possible that the reaction is catalytic: One gas appears in the liquid, and the other one exists only on the surface. However, this possibility can be ruled out as we observed the process for different electrode materials. Moreover, when the current is off $|\Delta d(t)|$ decreases linearly with time. A catalytic reaction would die faster (during one period) because no new adsorbed atoms appear on the surface. 

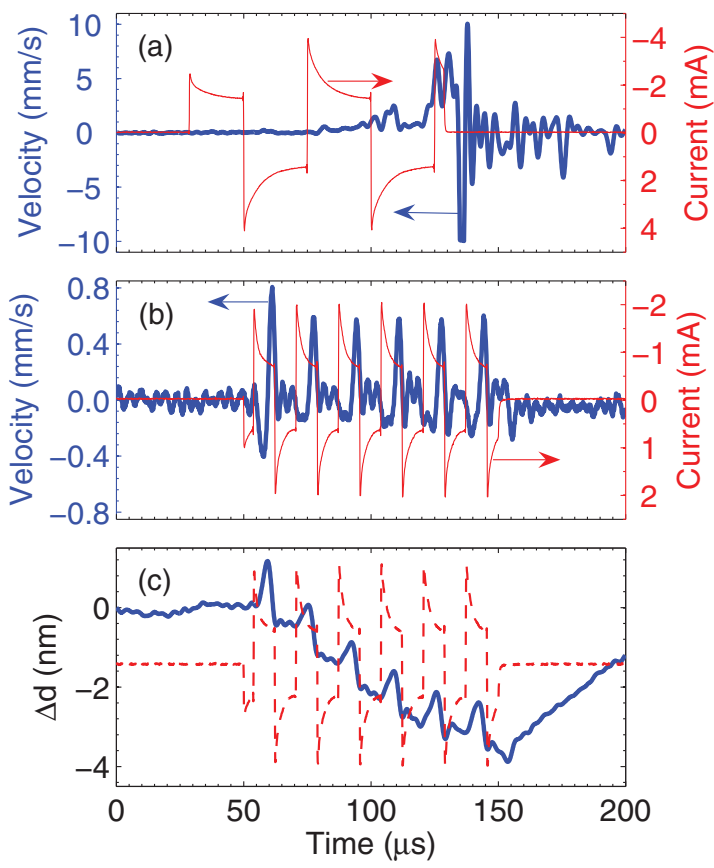

FIG. 3. (Color online) Observation with a vibrometer, $U=$ 4.5 V. (a) Processing at low frequency, $f=20 \mathrm{kHz}$, results in the formation of bubbles comparable in size with $\lambda / \pi$, which scatter light significantly. (b) The vibrometer signal and current as functions of time at $f=60 \mathrm{kHz}$. The thick curve [blue (black)] is the signal, and the thin curve [red (gray)] is the current. The signal peaks are clearly related to the current pulses. (c) The signal in (b) integrated in time (thick blue). The dashed curve (current, red) is given for eye guidance. The peaks in $\Delta d(t)$ indicate presence of a reaction between gases.

If the reaction is not included in the consideration, then, for alternating square pulses, the signal can be expressed via the current density as [12]

$$
v(t)=-\frac{\mathrm{d} \Delta d}{\mathrm{~d} t} \approx \frac{0.35 j_{F}}{4 e N_{\mathrm{sol}}} g(t), g(t)=\left\{\begin{array}{l}
2,0<t<T / 2 \\
1, T / 2<t<T
\end{array}\right.
$$

where $e$ is the absolute value of the electron charge, $N_{\text {sol }}$ is the concentration of the solution, and $j_{F}$ is the amplitude of the Faraday current density. Interaction of gases can make the right-hand side of Eq. (1) only smaller. Thus, we can use Eq. (1) to get a restriction on the current density. For maximal velocity $v_{\max }=0.6 \mathrm{~mm} / \mathrm{s}$ one finds $j_{F}>1900 \mathrm{~A} / \mathrm{cm}^{2}$. This is a very high value in comparison with the conventional (long-time) electrolysis [17], where $j_{F} \sim 1 \mathrm{~A} / \mathrm{cm}^{2}$. Using this value we can estimate the concentration of hydrogen near the electrode as $N \sim j_{F} T / 4 e(D T / 2)^{1 / 2}$, where $D$ is the gas diffusion coefficient. Taking the ratio with the saturated concentration $\left(N_{s}=4.7 \times 10^{17} \mathrm{~cm}^{-3}\right.$ for $\left.\mathrm{H}_{2}\right)$, one finds the level of relative supersaturation $S=N / N_{s}$ for $\mathrm{H}_{2}$ as 5000 and, similarly, for $\mathrm{O}_{2}$ one finds $S \sim 2000$. Usually a supersaturation of 100 is considered as a maximal [18] due to formation of a continuous gas film above the electrode. In our case it does not happen because the bubbles are very small on a microsecond time scale. Indeed, these high values of supersaturation guarantee homogeneous nucleation of bubbles.
All the observed phenomena can be understood as a chemical reaction that occurs in nanobubbles. Hydrogen produced by a negative voltage pulse creates high supersaturation in the liquid. This results in homogeneous nucleation of $\mathrm{H}_{2}$ bubbles that immediately start to grow. The positive half of the pulse produces oxygen, which diffuses into the $\mathrm{H}_{2}$ bubbles. For high frequencies, the gas composition in a bubble becomes stoichiometric while its size is far below $1 \mu \mathrm{m}$. The capillary pressure in bubbles (28 bar for a bubble $100 \mathrm{~nm}$ in diameter) promotes ignition of the reaction. The precise mechanism of this promotion is not yet clear, but the chemical equilibrium in bubbles is shifted [19], and it is known that fast dynamical processes play role for ignition on the macroscale [20]. If the frequency is low, the bubble reaches the stoichiometric composition for the larger size, and the capillary pressure is not sufficient to ignite the reaction. The critical diameter of a bubble is estimated as $d_{c}=100-200 \mathrm{~nm}$ [12]. The reaction manifests itself in $\Delta d(t)$ as periodic peaks but, when the current is off, the bubble formation and disintegration still goes on as long as supersaturation is not depleted. This explains the linear decrease in $|\Delta d(t)|$. The reaction in a bubble is accompanied by the release of a considerable amount of
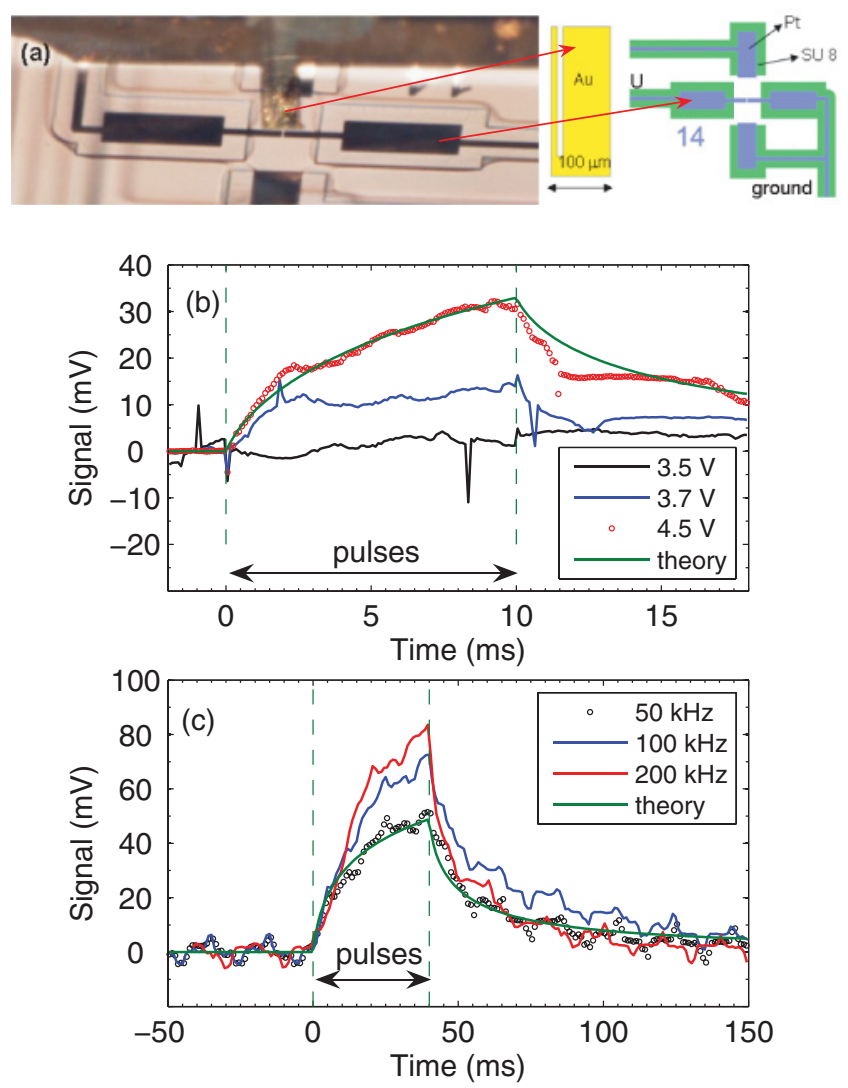

FIG. 4. (Color) Heating of the solution due to chemical reaction. (a) The gold probe (200 $\mathrm{nm}$ Au on a thing SiN membrane) positioned nearby the electrodes (angle of view $45^{\circ}$ ). Schematic views of the probe and electrodes are shown on the right. (b) Voltage on the probe as a function of time. Voltage pulses of different amplitude and $f=$ $100 \mathrm{kHz}$ were applied to the electrodes during $10 \mathrm{~ms}$. This figure demonstrates presence of the threshold in the heating. (c) Signal on the probe for pulses of different frequencies with $U=5 \mathrm{~V}$ and duration $40 \mathrm{~ms}$. The main component of the noise is $50 \mathrm{~Hz}$. 
chemical energy, fast condensation of the reaction product, and fast change in the pressure. As a result the bubble is destroyed in a violent act, but the details are not yet clear. The violent evolution of bubbles is the reason for the visible damage of the electrode surfaces.

We tried to observe the released energy in the visible light as expected for bulk combustion. With the low-light Andor iXon +855 EMCCD camera no signal was found. It has to be stressed that in a small volume the reaction can proceed very differently from the classical combustion. This is because the bubble size is much smaller than the reaction zone size $(\sim 10 \mu \mathrm{m})$ in the combustion theory [21].

The released energy must increase the temperature of the surrounding liquid. For our system the effect is small but still detectable [12]. We measured the temperature increase using a thin gold probe [22] positioned near the electrodes perpendicular to the sample surface [see Fig. 4(a)]. The heat produced by the reaction and the Joule heating of liquid change the resistance of the probe resulting in variation of voltage, which is measured (the current through the probe was $10 \mathrm{~mA}$ ). To minimize the cross-talk with the voltage pulses between electrodes, special care was taken to ensure that the probe is at the floating potential. The signal was amplified $10^{4}$ times and averaged over the time much larger than the period $T=1 / f$. Figure 4(b) shows the signal as a function of time for different voltages on the working electrode. In Fig. 4(c) the signal is presented for different frequencies. The threshold behavior visible in Fig. 4(b) and signal increase with frequency in Fig. 4(c) ensures that we observe the heating due to the reaction but not the Joule heating. Moreover, the magnitude of the signal due to the Joule heating is small according to our estimate [12].

The thermal problem was modeled to compare to the observations. The chemical reaction (happens in a very thin layer) can be considered as a surface heat source, and its magnitude can be expressed via the enthalpy of the reaction $\Delta H=-242 \mathrm{~kJ} / \mathrm{mol}$ and the number of reacting molecules estimated from the vibrometer measurements [12]. For $f=50 \mathrm{kHz}$ the local power per unit area was found to be $P_{s l}=0.4 \mathrm{MW} / \mathrm{m}^{2}$ (the largest value we observed was $1.3 \mathrm{MW} / \mathrm{m}^{2}$ ). However, the thermal problem depends on $P_{s l}$ averaged over the electrode area, which is less defined but was estimated as $P_{s} \approx 0.08 \mathrm{MW} / \mathrm{m}^{2}$ [12]. Heating of the liquid with a rectangular surface source (electrodes) was found analytically. The theoretical curves are in good agreement with the observations as one can see in Fig. 4(b) and 4(c) although we had to adjust slightly (within 30\%) the magnitude (but not the shape) of the signal due to uncertainty in $P_{s}$.

In this work we have provided evidence that chemical reaction between $\mathrm{H}_{2}$ and $\mathrm{O}_{2}$ gases can be ignited spontaneously in nanobubbles. Our finding can have a wide range of applications including fast reversible phase transitions, local delivery of a controllable amount of energy, or nanostructured combustion microengines, to name a few.

We thank D. Lohse, A. Prosperetti, J. Seddon, and L. Lefferts for discussions and K. Ma, P. van Zwol, M. Siekman, and $\mathrm{H}$. van Wolferen for technical assistance. This work was partially supported by the Dutch Technological Foundation (STW).
[1] G. Hodes, Adv. Mater. 19, 639 (2007).

[2] Q. S. Mei and K. Lu, Prog. Mater. Sci. 52, 1175 (2007).

[3] J. R. T. Seddon, E. S. Kooij, B. Poelsema, H. J. W. Zandvliet, and D. Lohse, Phys. Rev. Lett. 106, 056101 (2011).

[4] S.-T. Lou et al., J. Vac. Sci. Technol. B 18, 2573 (2000); M. Switkes and J. W. Ruberti, Appl. Phys. Lett. 84, 4759 (2004); R. Steitz et al., Langmuir 19, 2409 (2003).

[5] K. S. Suslick and D. J. Flannigan, Annu. Rev. Phys. Chem. 59, 659 (2008).

[6] K. S. Suslick, M. M. Mdleleni, and J. T. Reis, J. Am. Chem. Soc. 119, 9307 (1997).

[7] D. H. Lee, D. E. Pack, J. B. Yoon, S. Kwon, and E. Yoon, J. Micromech. Microeng. 12, 26 (2002); J. Peirs, D. Reynaerts, and F. Verolaetsen, Sens. Actuators A 113, 86 (2004).

[8] D. M. van den Broek and M. Elwenspoek, J. Micromech. Microeng. 18, 064003 (2008).

[9] D. A. Ateya, A. A. Shah, and S. Z. Hua, Rev. Sci. Instrum. 75, 915 (2004).

[10] S. Z. Hua, F. Sachs, D. X. Yang, and H. D. Chopra, Anal. Chem. 74, 6392 (2002).

[11] N. P. Brandon and G. H. Kelsall, J. Appl. Electrochem. 15, 475 (1985).
[12] See Supplemental Material at http://link.aps.org/supplemental/ 10.1103/PhysRevE.84.035302 for estimates and additional figures.

[13] P. G. Debenedetti, Metastable Liquids: Concept and Principles (Princeton University Press, Princeton, NJ, 1996).

[14] S. Yang et al., Langmuir 25, 1466 (2009).

[15] R. S. Taylor and C. J. Hnatovsky, J. Appl. Phys. 95, 8444 (2004).

[16] C. R. Neagu, J. G. E. Gardeniers, M. Elwenspoek, and J. J. Kelly, J. Micromech. Syst. 5, 2 (1996); C. G. Cameron and M. S. Freud, Proc. Natl. Acad. Sci. USA 99, 7827 (2002); D. D. Meng and C.-J. Kim, Lab Chip 8, 958 (2008).

[17] O. Teschke, D. M. Soares, and C. A. P. Evora, J. Appl. Electrochem. 13, 371 (1983); J. Krist'al, R. Kodym, K. Bouzek, and V. Jiricny, Electrochem. Comm. 10, 204 (2008).

[18] H. Vogt, J. Appl. Electrochem. 23, 1323 (1993).

[19] A. Sanfeld and A. Steinchen, Surf. Sci. 463, 157 (2000).

[20] V. V. Golub et al., J. Loss Prevent Process Ind. 20, 439 (2007); F. L. Dryer et al., Combust. Sci. Technol. 179, 663 (2007).

[21] B. Lewis and G. von Elbe, Combustion, Flames and Explosions of Gases (Academic Press, New York, 1987).

[22] J. Haneveld et al., J. Micromech. Microeng. 20, 125001 (2010). 